\title{
CT Angiography is Cost-Effective for Confirmation of Internal Carotid Artery Occlusions
}

\author{
Devin L. Brown, MD, MS, Stuart N. Hoffman, DO, Teresa L. Jacobs, MD, Kirsten L. Gruis, MD, MS, \\ Susan L. Johnson, MD, MS, Michael E. Chernew, PhD \\ From the Stroke Program, University of Michigan, Ann Arbor, MI (DLB, TLJ); Department of Neurology, Geisinger Medical Center, Danville, PA (SNH); Department of Neurology, \\ University of Michigan, Ann Arbor, MI (KLG); Department of Internal Medicine, University of Michigan, Ann Arbor, MI (SLJ); and Department of Health Care Policy, Harvard
} Medical School, Boston, MA (MEC)

\section{A B S T R A C T}

\section{BACKGROUND AND PURPOSE}

While sensitive to internal carotid artery (ICA) occlusion, carotid ultrasound can produce false-positive results. CT angiography (CTA) has a high specificity for ICA occlusion and is safer and cheaper than catheter angiography, although less accurate. We determined the cost-effectiveness of CTA versus catheter angiography for confirming an ICA occlusion first suggested by carotid ultrasound.

\section{METHODS}

A Markov decision-analytic model was constructed to estimate the cost-effectiveness of CTA compared with catheter angiography in a hypothetical cohort of symptomatic patients with a screening examination consistent with an ICA occlusion. Costs in 2004 dollars were estimated from Medicare reimbursement. Effectiveness was measured in quality-adjusted life years.

\section{RESULTS}

The 2-year cost in the CTA scenario was $\$ 9,178$, and for catheter angiography, $\$ 11,531$, consistent with a $\$ 2,353$ cost-savings per person for CTA. CTA resulted in accrual of 1.83 quality-adjusted life years while catheter angiography resulted in 1.82 quality-adjusted life years. CTA was less costly and marginally more effective than catheter angiography. In sensitivity analyses, when CTA sensitivity and specificity were allowed to vary across a plausible range, CTA remained cost-effective.

\section{CONCLUSIONS}

After screening examination has suggested an ICA occlusion, confirmatory testing with CTA provides similar effectiveness to catheter angiography and is less costly.
Keywords: Cerebral angiography, cost-benefit analysis, health services research.

Acceptance: Received September 7, 2007, and in revised form September 7 2007. Accepted for publication October 5, 2007.

Correspondence: Address correspondence to Devin L. Brown, MD, MS The Cardiovascular Center-Stroke Program, 1500 E. Medical Center Drive, SPC\#5855, Ann Arbor, Ml 48109-5855. E-mail: devinb@umich.edu.

$\mathrm{J}$ Neuroimaging 2008;18:355-359.

DOI: $10.1111 / j .1552-6569.2007 .00216 . x$

\section{Introduction}

Carotid endarterectomy (CEA) is superior to medical therapy in prevention of stroke in patients with symptomatic severe carotid artery disease. ${ }^{1,2}$ Initial screening for carotid disease in stroke patients is commonly performed with carotid ultrasound (CUS). ${ }^{3}$ In $15 \%$ of symptomatic patients, CUS suggests an internal carotid artery (ICA) occlusion. However, because of technical limitations of CUS, the artery is actually patent in up to $14 \%$ of these cases. ${ }^{4}$ Therefore, when a CUS suggests an ICA occlusion, further testing is often deemed necessary.

The second test selected for confirmation of a suspected occlusion is often intra-arterial conventional angiography (CA) or CT angiography (CTA). These two tests, CA and CTA are not as flow-dependent as CUS and MR angiography (MRA), ${ }^{5-7}$ and therefore are more accurate in differentiating between a complete occlusion and severe stenosis. ${ }^{8,9} \mathrm{CA}$ is considered the reference standard for assessing the degree of ICA stenosis. CA carries with it a risk of stroke and is expensive, while
CTA is safer and less costly than CA, but is less accurate. We therefore performed a cost-utility analysis to determine the costeffectiveness of using CTA compared with CA to confirm a suspected ICA occlusion.

\section{Methods \\ Model}

A decision analytic model was constructed to consider the use of two alternative strategies, CTA and CA, to confirm the diagnosis of an ICA occlusion suspected based on CUS in a hypothetical cohort of symptomatic patients. Four possible outcomes were considered: death from the diagnostic test, stroke from the diagnostic test, stroke by 2 years, and death by 2 years. Two-years was selected for the time-horizon as the risk of stroke for those with a severe stenosis who undergo CEA approximates those treated medically after $2-3$ years. ${ }^{10}$ Furthermore, estimates of probabilities beyond 2 years are less 


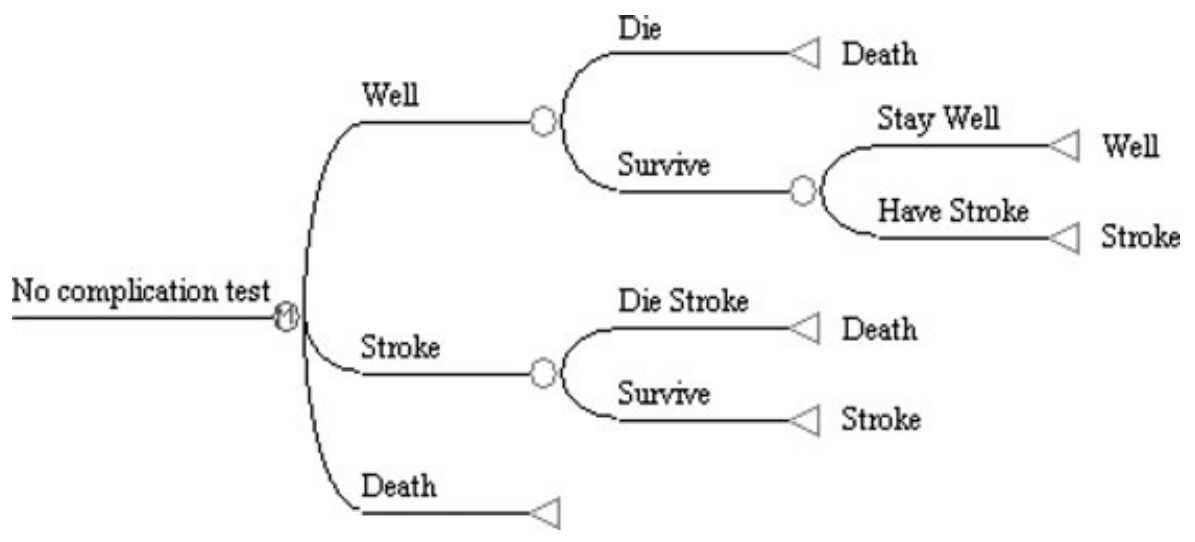

Fig 1. Markov model.

well-established. Long-term outcomes were modeled using Markov models. Markov processes use simulation techniques to evaluate a hypothetical cohort of patients' prognoses given repeated exposure to a particular health risk. Each hypothetical patient who did not have a diagnostic test-related stroke was modeled to start in the well state. $\mathrm{He} /$ she could transition from well to stroke, or dead as dictated by monthly Markov transition probabilities. A diagram of the Markov model in isolation is shown in Figure 1. Model inputs were derived from the literature. All estimates derived from the literature were ex- plored in a one-way sensitivity analysis across a plausible range of values, as shown in Table 1. Two-way sensitivity analyses were also conducted with both CTA sensitivity and specificity and several key variables: probability of ICA occlusion at the time of the test, probability of stroke from CA, and the utility of stroke. A "worst-case" scenario analysis was then performed biasing the analysis against CTA. This used the costs, sensitivities, specificities, and complications found in Table 1, which were favoring CA and disadvantaging CTA: the lowest values for CTA sensitivity and specificity were used, while the highest costs and

Table 1. List of Probabilities, Utilities, and Costs for the Reference Cases, and Ranges Used for Sensitivity Analyses

\begin{tabular}{|c|c|c|c|}
\hline & Reference Case & Lower Range Tested & Upper Range Tested \\
\hline Probability of ICA occlusion & $.89(33)$ & $.86(4)$ & $.97(11)$ \\
\hline CTA sensitivity & $.97(13)$ & $.92(13)$ & $1.0(13)$ \\
\hline CTA specificity & $.99(13)$ & $.98(13)$ & $1.0(13)$ \\
\hline Stroke CTA & 0 & - & - \\
\hline CA sensitivity and specificity & 1.0 & - & - \\
\hline Death CTA & $.000006(16)$ & $.000004(35)$ & .0005 \\
\hline Stroke CA & $.01(15)$ & $.0014(36)$ & .015 \\
\hline Death CA & $.0006(15,36)$ & .0002 & .0010 \\
\hline Monthly probability of death after ICA occlusion & $.0056(14)$ & .004 & .007 \\
\hline Monthly probability of death $70-99 \%$ stenosis & $.0048(1)$ & .003 & .006 \\
\hline Monthly probability of stroke after ICA occlusion & $.0048(14)$ & .003 & .006 \\
\hline Monthly probability of stroke after ICA occlusion with CEA & $.0053(14)$ & .004 & .007 \\
\hline Monthly probability of stroke $70-99 \%$ stenosis with CEA & $.0056(1)$ & .004 & .02 \\
\hline Monthly probability of stroke $70-99 \%$ stenosis without CEA & $.013(1)$ & .005 & .02 \\
\hline Utility of stroke state & $.6(17)$ & $.0(17)$ & $.7(17)$ \\
\hline Cost CEA & $\$ 16,304(37)$ & $\$ 10,768(18)$ & $\$ 20,000$ \\
\hline Cost CTA & $\$ 842(38,39)$ & $\$ 500$ & $\$ 1,500$ \\
\hline Cost CA & $\$ 3,042(38,39)$ & $\$ 2,000$ & $\$ 4,000$ \\
\hline Cost stroke death & $\$ 19,295(19,20)$ & $\$ 10,000$ & $\$ 30,000$ \\
\hline Cost of stroke hospitalization & $\$ 10,726(19)$ & $\$ 5000$ & $\$ 15,000$ \\
\hline Monthly cost of stroke & $\$ 2,170(20)$ & $\$ 1,000$ & $\$ 3,000$ \\
\hline Cost of death from CTA & $\$ 5,000^{*}$ & $\$ 2,500$ & $\$ 10,000$ \\
\hline
\end{tabular}

*Estimated.

Internal carotid artery (ICA).

CT angiogram (CTA).

Conventional angiogram (CA).

Carotid endarterectomy (CEA). 
complications for CTA and the lowest values for complications and costs for CA were used. We further explored the effect of biasing variables on the model results by only partially biasing the analysis against CTA. First all CTA variables that disadvantaged CTA were used. Separately we used all CA variables that favored CA. As contrast-enhanced MRA is often used as a screening test for ICA disease, the analysis was repeated substituting the accuracy of contrast-enhanced MRA for that of CUS. The decision tree was analyzed using Data 4.0 (TreeAge software, Inc, Williamstown, MA).

\section{Baseline Probability of ICA Occlusion}

The positive predictive value of carotid ultrasound in detecting a complete ICA occlusion was estimated to be $.97 .{ }^{11}$ This value was used for the true baseline estimate of ICA occlusion in those undergoing CA or CTA. The analysis was repeated assuming contrast-enhanced MRA was used to screen individuals for carotid disease using a baseline prevalence equal to the positive predictive value of contrast-enhanced MRA for ICA occlusion, $.93 .{ }^{12}$ The value of the probability of occlusion was allowed to vary in sensitivity analyses (see Table 1 ). It was assumed that a $0-69 \%$ stenosis and complete occlusion are never mistaken for one another by any of the diagnostic tests.

\section{Accuracy of CTA}

Koelemay et al. ${ }^{13}$ systematically reviewed the literature analyzing the aggregated sensitivity and specificity of CTA. Sensitivity and specificity values were obtained from this study and were allowed to vary in sensitivity analysis through the range of their $99 \%$ confidence intervals.

\section{Risks}

Estimates of stroke and mortality probabilities can be found in Table 1. Monthly probabilities of stroke were estimated from the North American Symptomatic Carotid Endarterectomy Trial (NASCET) ${ }^{1}$ for patients with 70-99\% stenoses (incorporating strokes and deaths as a result of CEA where applicable), and from an analysis of prospective and retrospective series for those with complete occlusions. ${ }^{14}$ Two-year mortality for CEA and non-CEA groups was not significantly different in the NASCET study and thus were averaged together. It was assumed that there was no benefit conferred by CEA in patients with complete occlusions and that this surgery would yield a $1 \%$ higher stroke risk above baseline when performed in those with a complete occlusion.

For the reference case analysis, a $1 \%$ stroke risk and a $.06 \%$ death risk associated with CA were estimated from data pooled from eight prospective studies. ${ }^{15}$ It was assumed based on the literature that the only significant complication from CTA was death related to contrast administration. This was estimated to occur in $.0006 \%$ of contrast injections. ${ }^{16}$

\section{Outcomes}

Utilities are measures between 0 , representing death, and 1, representing perfect health, which can be assigned to a health state in order to account for the relative adverse effect the condition has on quality of life. Quality-adjusted life years (QALYs) were calculated by multiplying utilities by life-years spent in that health state. Utility measures were estimated from the literature. The utility value used for stroke, .6, was obtained from healthy subjects using the standard gamble approach, ${ }^{17}$ but this value was allowed to vary widely in sensitivity analyses. ${ }^{17}$

\section{Costs}

A societal perspective, which considers costs regardless of the identity of the payer, was taken. All costs were converted to 2004 US dollars and are found in Table 1. The cost of CA and CTA were estimated from Medicare fee schedules and included technical and physician components. The cost of CEA, monthly cost of stroke, and cost of death from stroke were estimated from studies where Medicare reimbursement was used. ${ }^{18-20}$ Costs, calculated using the cost-to-charge ratio or Medicare allowable payments, included hospitalization, physician time, rehabilitation, durable medical equipment, and skilled nursing home care. ${ }^{20}$ Nonskilled nursing home care and outpatient medications were also considered. ${ }^{20}$

\section{Results}

The average patient undergoing CTA experienced 1.83 QALYs at a cost of $\$ 9,178$; while those undergoing CA experienced 1.82 QALYs at a cost of $\$ 11,531$, resulting in an incremental cost of $\$ 2,353$ for CA. The cost-effectiveness of CTA was $\$ 5,012$ and for CA was $\$ 6,322$. Repeating the analysis assuming that contrast-enhanced MRA was used for screening yielded similar results: CTA remained dominant over CA. Each variable was allowed to vary in one-way sensitivity analysis using the value ranges found in Table 1 . The results of the analysis were insensitive to these alterations, with the CTA strategy remaining dominant over CA. Two-way sensitivity analyses performed with CTA sensitivity and specificity and the key variables (probability of ICA occlusion at the time of the test, probability of stroke from CA, and the utility of stroke) also supported CTA as the preferred strategy throughout the ranges tested.

In the "worst case" scenario model, where the costs, sensitivities, specificities, and complications were all biased against CTA and in favor of CA, the incremental cost-effectiveness of CTA was $\$ 690,225$ suggesting that CTA was no longer costeffective compared with CA. CTA remained dominant over $\mathrm{CA}$ in the two partially biased models.

\section{Discussion}

This cost-effectiveness analysis suggests that CTA is preferable to CA to confirm a symptomatic carotid occlusion suspected based on a screening ultrasound or MRA. Despite the superior sensitivity and specificity of CA in the model compared with CTA, the greater cost of CA along with its higher rates of complications shifts the advantage to CTA. In the scenario with all model input variables biased against CTA and in favor of CA, the incremental cost-effectiveness of CTA was no longer in a range considered to be cost-effective. ${ }^{21}$ The decision to pursue further diagnostic testing in a patient who is suspected of having an ICA occlusion is a tradeoff between potential complications of the confirmatory test and the need to identify an operable ICA stenosis. Despite CTA's inferior accuracy, its lower complication rate and lower cost compared with 
CA resulted in a favorable cost-effectiveness ratio in almost all scenarios tested.

For each 100 patients who receive a CTA in place of a CA for evaluation of a carotid occlusion, over $\$ 235,000$ is saved. Although the difference in total costs between the tests is quite large, the difference in outcomes for the two diagnostic strategies compared in this analysis is small, suggesting that the model results are largely being driven by the relative costs rather than the relative effectiveness. Nonetheless, sensitivity analyses involving both cost and effectiveness variables show that alterations in key probabilities across plausible ranges do not alter the superior cost-effectiveness of CTA in one- and two-way sensitivity analyses, supporting the robustness of the model. Furthermore, omitting CA-related TIA, hematoma, and infection from the model biased the analysis in favor of CA. If these unmodeled variables were to be considered, in addition to patient preferences, the support in favor of CTA would become even stronger. ${ }^{22}$ Use of a two-year time horizon, rather than modeling until death, also biased the model in favor of CA. Despite these numerous biases in favor of CA, CTA remained cost-effective compared with CA through all scenarios tested.

Previous cost-effectiveness analyses related to carotid disease have not considered CTA. ${ }^{23-26}$ Furthermore, these studies have dealt with overall strategies for screening for surgical ICA lesions, rather than addressing more focused questions such as the current analysis. Although CTA has limitations, such as differentiating between a moderate and severe stenosis, ${ }^{27,28}$ there have been many series showing that it is an accurate technology for detecting occlusions. ${ }^{8,9,13}$ When selecting a test for carotid artery evaluation, it is important to select the test based on the specific question being asked.

A recent post-hoc meta-analysis suggested that endarterectomy for the subset of severe stenoses regarded as nearocclusions may not be beneficial when assessing 5- and 8-year outcomes. $^{29,30}$ The validity of these types of subgroup analyses and the statistical methods used are questionable. ${ }^{31}$ Nonetheless, the benefit measured at 1 and 3 years post-CEA in those with near-occlusions is better established. ${ }^{30,32}$ Although there is evidence that not all false-positive occlusions detected by CUS or contrast-enhanced MRA can be attributed to nearocclusions, ${ }^{4-6,33}$ the true proportion is unknown. Sensitivity analysis addressed the impact of altering the benefit of CEA in the severe stenosis group. As the monthly probability of stroke in those who received CEA approached those who did not, CTA remained more cost-effective than CA.

The true accuracy of CTA in detecting carotid artery occlusions is unknown. Studies reporting its accuracy are likely from centers with significant experience with CTA. The accuracy of CTA in less experienced hands may not be so high, which may limit the applicability of our results to centers proficient in CTA performance and interpretation. The sensitivity and specificity of CTA conditional on the ultrasound results may also differ from the primary sensitivity and specificity of CTA. The estimates of CTA sensitivity and specificity reported in the literature may be subject to publication bias; ${ }^{34}$ however, the concordance among many studies argues against this being a major factor. As with any cost-utility analysis, the current results are limited by the accuracy of the estimates used in the model.
To mitigate against this, we performed multiple sensitivity analyses across a wide range of values, and included a "worst-case" scenario model.

\section{Conclusion}

Given rising health care costs, cost-minimizing diagnostic strategies need to be explored. This analysis suggests that CTA can be used as a less expensive alternative to CA for confirmation of a suspected ICA occlusion, without sacrificing patient outcome.

Dr. Brown is supported by an NINDS Career Development Award (K23 NS051202); Dr. Gruis is supported by an NINDS Career Development Award (K23 NS055200).

\section{References}

1. North American Symptomatic Carotid Endarterectomy Trial Collaborators. Beneficial effect of carotid arterectomy in symptomatic patients with high-grade carotid stenosis. $N$ Engl J Med 1991;325:445-453.

2. Randomised trial of endarterectomy for recently symptomatic carotid stenosis: final results of the MRC European Carotid Surgery Trial (ECST). Lancet 1998;351:1379-1387.

3. Goldstein LB, Bonito AJ, Matchar DB, et al. US national survey of physician practices for the secondary and tertiary prevention of ischemic stroke. Design, service availability, and common practices. Stroke 1995;26:1607-1615.

4. Bridgers S. Clinical correlates of Doppler/ultrasound errors in the detection of internal carotid artery occlusion. Stroke 1989;20:612615 .

5. Cosottini M, Pingitore A, Puglioli M, et al. Contrast-enhanced threedimensional magnetic resonance angiography of atherosclerotic internal carotid stenosis as the noninvasive imaging modality in revascularization decision making. Stroke 2003;34:660-664.

6. Kollias S, Binkert C, Ruesch S, Valavanis A. Contrast-enhanced MR angiography of the supra-aortic vessels in 24 seconds: a feasibility study. Neuroradiology 1999;41:391-400.

7. Wikström J, Johansson L, Rossitti S, Karacagil S, Ahlström H. High-resolution carotid artery MRA: comparison with fast dynamic acquisition and duplex ultrasound scanning. Acta Radiologica 2002; 43:256-261.

8. Chen CJ, Lee TH, Hsu HL, et al. Multi-Slice CT angiography in diagnosing total versus near occlusions of the internal carotid artery: comparison with catheter angiography. Stroke 2004;35:8385.

9. Sameshima T, Futami S, Morita Y, et al. Clinical usefulness of and problems with three-dimensional CT angiography for the evaluation of arteriosclerotic stenosis of the carotid artery: comparison with conventional angiography, MRA, and ultrasound sonography. Surg Neurol 1999;51:301-308.

10. Barnett H, Taylor D, Eliasziw M, et al. Benefit of carotid endarterectomy in patients with symptomatic moderate or severe stenosis. $N$ EnglJ Med 1998;339:1415-1425.

11. Nederkoorn P, Mali W, Eikelboom B, et al. Preoperative diagnosis of carotid artery stenosis: accuracy of noninvasive testing. Stroke 2002;33:2003-2008.

12. Remonda L, Senn P, Barth A, Arnold M, Lovblad K, Schroth G. Contrast-enhanced 3D MR angiography of the carotid artery: comparison with conventional digital subtraction angiography. AJNR Am J Neuroradiol 2002;23:213-219.

13. Koelemay MJW, Nederkoorn PJ, Reitsma JB, Majoie CB. Systematic review of computed tomographic angiography for assessment of carotid artery disease. Stroke 2004;35:2306-2312. 
14. Klijn CJ, Kappelle LJ, Tulleken CA, van Gijn J. Symptomatic carotid artery occlusion. A reappraisal of hemodynamic factors. Stroke 1997;28:2084-2093.

15. Hankey G, Warlow C, Sellar RJ. Cerebral angiographic risk in mild cerebrovascular disease. Stroke 1990;21:209-222.

16. Katayama H, Yamaguchi K, Kozuka T, Takashima T, Seez P, Matsuura $\mathrm{K}$. Adverse reactions to ionic and nonionic contrast media. A report from the Japanese Committee on the Safety of Contrast Media. Radiology 1990;175:621-628.

17. Post P, Stiggelbout A, Wakker PP. The utility of health states after stroke: a systematic review of the literature. Stroke 2001;32:14251429.

18. Cronenwett J, Birkmeyer J, Nackman G, et al. Cost-effectiveness of carotid endarterectomy in asymptomatic patients. J Vasc Surg 1997;25:298-311.

19. Holloway RG, Witter DM, Jr, Lawton KB, Lipscomb J, Samsa G. Inpatient costs of specific cerebrovascular events at five academic medical centers. Neurology 1996;46:854-860.

20. Samsa GP, Bian J, Lipscomb J, Matchar DB. Epidemiology of recurrent cerebral infarction: a medicare claims-based comparison of first and recurrent strokes on 2-year survival and cost. Stroke 1999;30:338-349.

21. Ubel PA, Hirth RA, Chernew ME, Fendrick AM. What is the price of life and why doesn't it increase at the rate of inflation? Arch Intern Med 2003;163:1637-1641.

22. Patel SG, Collie DA, Wardlaw JM, et al. Outcome, observer reliability, and patient preferences if CTA, MRA, or Doppler ultrasound were used, individually or together, instead of digital subtraction angiography before carotid endarterectomy. J Neurol Neurosurg Psychiatry 2002;73:21-28.

23. Benade MM, Warlow CP. Costs and benefits of carotid endarterectomy and associated preoperative arterial imaging: a systematic review of health economic literature. Stroke 2002;33:629-638.

24. Buskens E, Nederkoorn PJ, Buijs-van der Woude T, et al. Imaging of carotid arteries in symptomatic patients: cost-effectiveness of diagnostic strategies. Radiology 2004;233:101-112.

25. Kent KC, Kuntz KM, Patel MR, et al. Perioperative imaging strategies for carotid endarterectomy. An analysis of morbidity and costeffectiveness in symptomatic patients. JAMA 1995;274:888-893.
26. King-Im JM, Hollingworth W, Trivedi RA, et al. Cost-effectiveness of diagnostic strategies prior to carotid endarterectomy. Ann Neurol 2005;58:506-515.

27. Anderson G, Ashforth R, Steinke D, Ferdinandy R, Findlay J. CT angiography for the detection and characterization of carotid artery bifurcation disease. Stroke 2000;31:2168-2174.

28. Josephson SA, Bryant SO, Mak HK, Johnston SC, Dillon WP, Smith WS. Evaluation of carotid stenosis using CT angiography in the initial evaluation of stroke and TIA. Neurology 2004;63:457-460.

29. Rothwell P, Gutnikov S, Warlow CP. Reanalysis of the final results of the European Carotid Surgery Trial. Stroke 2003;34:514-523.

30. Rothwell P, Eliasziw M, Gutnikov S, et al. Analysis of pooled data from the randomized controlled trials of endarterectomy for symptomatic carotid stenosis. Lancet 2003;361:107-116.

31. Brookes ST, Whitely E, Egger M, Smith GD, Mulheran PA, Peters TJ. Subgroup analyses in randomized trials: risks of subgroupspecific analyses; power and sample size for the interaction test. $J$ Clin Epidemiol 2004;57:229-236.

32. Morgenstern L, Fox A, Sharpe B, Eliasziw M, Barnett H, Grotta JC. The risks and benefits of carotid endarterectomy in patients with near occlusion of the carotid artery. Neurology 1997;48:911-915.

33. Bornstein N, Beloev Z, Norris J. The limitations of diagnosis of carotid occlusion by Doppler ultrasound. Ann Surg 1998;207:315317.

34. Begg C, Berlin J. Publication bias and dissemination of clinical research. J Natl Cancer Inst 1989;81:107-115.

35. Lasser EC, Lyon SG, Berry CC. Reports on contrast media reactions: analysis of data from reports to the U.S. Food and Drug Administration. Radiology 1997;203:605-610.

36. Kaufmann TJ, Huston J, Mandrekar JN, Schleck CD, Thielen KR, Kallmes DF. Complications of diagnostic cerebral angiography: evaluation of 19,826 consecutive patients. Radiology 2007;243:812819.

37. Nussbaum ES, Heros RC, Erickson DL. Cost-effectiveness of carotid endarterectomy. Neurosurgery 1996;38:237-244.

38. Center for Medicare and Medicaid Services. Fed Reg Depar Health Human Services 2004;69:836-838.

39. Centers for Medicare and Medicaid Services. Medicare Physician Fee Schedule. Michigan Locality 012004. 